\title{
Identification and Functional Evaluation of miR-4633-5p as a Biomarker and Tumor Suppressor in Metastatic Melanoma
}

\author{
Bingqing Zou Wenjia Zhu ${ }^{\mathrm{b}}$ Hongqin Liu ${ }^{\mathrm{b}}$ Shuyi Wang ${ }^{\mathrm{b}}$ Hongguang Zhu \\ aDepartment of Pathology, Shanghai Medical College, Fudan University, Shanghai, ${ }^{b}$ Department of \\ Pathology, Eye \& ENT Hospital, Fudan University, Shanghai, China
}

\section{Key Words}

Sinonasal mucosa $\cdot$ Melanoma $\cdot$ Head and neck $\cdot$ MicroRNA $\cdot$ Metastasis

\begin{abstract}
Background/Aims: Sinonasal mucosal melanoma (SMM) is a rare but extremely aggressive disease. Interestingly, however, as lethal as SMM, a few patients could survive for over 5 years without metastasis. However, biomarkers for metastatic SMM are lacking. Methods: Lasercapture microdissection combined with microRNA microarray and RT-qPCR was performed in formalin-fixed paraffin-embedded tissue samples from SMM patients whose follow-up studies were carried out in parallel. In vitro cell proliferation and invasion assays, gelatin zymography, western blot analysis and RT-qPCR were performed in melanoma cell lines. Results: In the discovery stage, miR-4633-5p expressed differentially in sinonasal mucosal melanoma patients with short and long disease-specific survival. Subsequent large-sample validation revealed that expression of miR-4633-5p was lower in metastatic SMM than in non-metastatic patients $(\mathrm{P}<0.001)$. Moreover, miR-4633-5p pow was able to identify metastatic SMM with specificity of $100 \%(5 / 5)$ and sensitivity of $87.5 \%$ (21/24). Multivariate analysis further pinpointed miR4633-5p as an independent marker for metastasis (relative risk: $54.22, \mathrm{P}<0.001$ ). In vitro, overexpression of miR-4633-5p suppressed the growth and invasiveness of melanoma cells through inhibiting activation of Akt pathway and secretion of MMP2, while knockdown of miR-4633-5p reversed the inhibitory effects. Conclusion: Our findings underpin miR-4633-5p as a predictive biomarker in metastatic SMM and a pivotal tumor suppressor that negatively regulates the invasive growth of melanoma cells. Quantitative detection of miR-4633-5p can diagnostically predict the risk of metastasis in SMM patients, which, in turn, may lead to more personalized treatment with better prognosis.




\section{Cellular Physiology Cell Physiol Biochem 2018;49:1364-1379

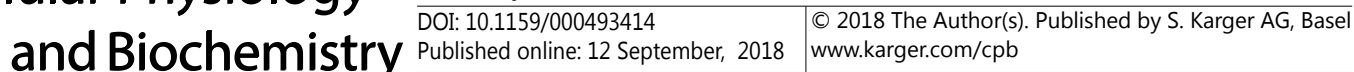

\section{Introduction}

Melanoma is a large entity of malignant tumor with increasing incidence and poor prognosis [1]. Among melanomas of all sites, mucosal melanoma takes up less than 1\%; among which, $1 / 3$ occurs in the sinonasal tract [2-4]. The extreme aggressiveness of sinonasal mucosal melanoma is gradually being acknowledged. From the $7^{\text {th }}$ edition of AJCC Cancer Staging Manual on, mucosal melanoma has been assigned its own staging system, which is distinct from that of other mucosal diseases and cutaneous melanoma [1]. Stages $\mathrm{T} 1$ and T2 were also abolished to warn clinicians that even shallow and confined head and neck mucosal melanoma is of poor prognosis [1]. Therefore, in the $7^{\text {th }}$ edition of AJCC Cancer Staging Manual, development of sinonasal mucosal melanoma was categorized into four stages, namely stages III, IVA, IVB and IVC. According to the $8^{\text {th }}$ edition of AJCC Cancer Staging Manual, 5-year survival rates of nasal and sinonasal mucosal melanoma were $15-30 \%$ and $0-5 \%$, respectively [1]. In our previous study, we also found that, as lethal as sinonasal melanoma, approximately $10 \%$ of patients could survive for over 5 years without metastasis. However, biomarker for metastatic sinonasal mucosal melanoma is lacking.

Quantitative detection of microRNAs (miRNAs) has been found to be useful in differential diagnosis, for the expression pattern of miRNAs is usually disease-specific [5-9]. As short, noncoding RNA, one striking feature of miRNA is its molecular stability that, unlike vulnerable long chain RNAs, miRNAs can be well preserved in formalin-fixed paraffin-embedded (FFPE) tissue over years, which renders miRNAs an excellent candidate for researches exploring biomarkers in particularly rare diseases such as sinonasal mucosal melanoma $[6,10,11]$.

Akt, also known as protein kinase B, is a serine/threonine kinase that promotes cell proliferation, cell cycle and survival [12]. Akt responds to numerous upstream signals by altering its cellular distribution and kinase activity, which subsequently leads to an altered phosphorylation status of its downstream targets. In melanoma, Akt was reported to promote tumor growth, metastasis and development of chemoresistance [13]. Furthermore, the phosphorylation status of Akt was also associated with activity of MMP2 (matrix metalloproteinase-2) in melanoma, which cooperatively expedites the invasion of melanoma cells and, thus, metastasis [14].

The present study aims to identify potential biomarkers in metastatic sinonasal mucosal melanoma. Using miRNA microarray combined with laser-capture microdissection, we found that miR-4633-5p expressed differentially in sinonasal mucosal melanoma patients with long and short disease-specific survival. Subsequent large-sample validation suggested that low expression of miR-4633-5p could accurately and independently discriminate metastatic sinonasal mucosal melanoma patients from non-metastatic patients. Additionally, miR4633-5p could functionally suppress the invasive growth of melanoma cells through the inhibition of MMP2 secretion and the inactivation of Akt pathway in vitro.

\section{Materials and Methods}

\section{Clinical specimens and follow-up study}

Primary human sinonasal mucosal melanoma samples were collected at the time of surgery (19982013) from patients enrolled in Eye, Ear, Nose and Throat Hospital affiliated to Fudan University, a tertiary referral hospital that serves patients across East China. Informed consents were obtained from all patients and approval acquired by the Ethics Committee of Eye, Ear, Nose and Throat Hospital affiliated to Fudan University. Patients were followed up in October, 2016. For the convenience of statistical analysis, all tumors were staged according to the $7^{\text {th }}$ edition of AJCC Cancer Staging Manual, which was replaced by a TNM staging system in the $8^{\text {th }}$ edition [14]. Mutational status of the clinical specimens was not available. 


\section{Cellular Physiology Cell Physiol Biochem 2018;49:1364-1379

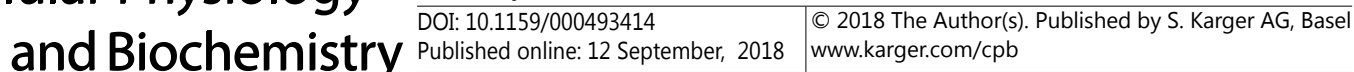

Zou et al.: miR-4633-5p in Metastatic Melanoma

\section{Split-sample method}

In order to find out metastasis-related miRNAs, all cases were divided into the following groups. Cases with disease-specific survival (DSS) $\leq 12$ months were defined as DSS-short. Cases with DSS $\geq 36$ months were defined as DSS-long. Cases that developed metastasis (either regional or distant) were classified into the metastatic group and those without clinical sign of metastasis until last follow-up were the nonmetastatic group. Of all the cases in the metastatic group, all but one case developed distant metastasis.

In all, 63 cases were collected and were reviewed to confirm diagnosis of primary sinonasal mucosal melanoma. Seven cases were excluded from further analysis. Four of them had insufficient archived tumor tissue and another three had heavy inflammation, rendering purity of melanoma cell $>85 \%$ unachievable. Upon strict crosschecking and pairing of available clinicopathologic parameters, 4 DSS-long cases and 4 DSS-short cases were matched and were chosen as the discovery cohort. The validation cohort consisted of 8 DSS-long cases and 8 DSS-short cases that were randomly chosen. The application cohort consisted of the rest 32 cases.

Laser-capture microdissection (LCM)

LCM was performed as previously described [6]. Briefly, $5 \mu$ m-thick FFPE sections were used for LCM. A hematoxylin and eosin-stained section was consulted in each case to guide the area of interest. Melanoma cells were captured using ultraviolet laser cutting following manufacturer's protocol to ensure purity of melanoma cell $>85 \%$.

\section{Microarray hybridization and data analysis}

The expression of 2549 human miRNAs (from Sanger miRBase V.21.0) in the discovery cohort was analyzed by Human microRNA Microarray (Agilent Technologies, Shanghai, China). Sample labeling, microarray hybridization and washing were performed according to manufacturer's protocols. Briefly, total RNA ( 100 ng) extracted from LCM-captured tumor tissue were dephosphorylated, denaturated, labeled with Cyanine-3-CTP and purified. The labeled RNAs were then hybridized onto the microarray. After washing, the microarray was scanned with the Agilent Scanner G2505C (Agilent Technologies).

The microarray image information was converted into raw data using Feature Extraction software (V.10.7.1.1, Agilent Technologies). After background subtraction, the signal was exported into the Genespring software (V.13.1, Agilent Technologies) for quantile normalization. In each pair of cases, differentially expressed miRNAs were defined as fold change $\geq 2$.

\section{RNA extraction and quality control}

Total RNA of FFPE sections and cultured cells were extracted using RecoverAll ${ }^{\mathrm{TM}}$ Total Nucleic Acid Isolation Kit for FFPE (AM1975, ThermoFisher Scientific, Shanghai, China) and TRIzol (15596026, ThermoFisher Scientific), respectively, following manufacturer's protocol. RNA samples were stored at -80?.

Concentration and quality of RNA was measured by NanoDrop 2000 (NanoDrop Technologies, Waltham, Massachusetts, USA). RNA samples were excluded from further analysis if OD 260/280<1.8.

\section{Reverse-transcriptase quantitative PCR (RT-qPCR)}

Quantification of miRNAs in the validation and the application cohorts were carried out using RTqPCR. Reverse transcription of miRNA and mRNA and quantitative PCR were carried out using Taqman microRNA Reverse Transcription Kit, High Capacity cDNA Reverse Transcription Kit and Taqman Universal PCR Master Mix and SYBR ${ }^{\mathrm{TM}}$ Green PCR Master Mix (Applied Biosystems, Shanghai, China) following manufacturer's protocols. Primers and probes of U6 small nuclear RNA (snRNA), miR-30c-2-3p, miR-4633$5 p$ and miR-4633-3p were purchased (Invitrogen, Shanghai, China). Primers of MMP2, MMP9 and GAPDH were synthesized (Sangon Biotech, Shanghai, China). Primer sequences of MMP2, MMP9 and GAPDH were retrieved from PrimerBank [15]. The expression of U6 snRNA and GAPDH was used as endogenous control for quantification of miRNA and mRNA, respectively. All experiments were carried out in 3 technical repeats and 3 biological repeats. 


\section{Cellular Physiology Cell Physiol Biochem 2018;49:1364-1379 \begin{tabular}{ll|l} 
and Biochemistry Published online: 12 September, 2018 & $\begin{array}{l}\text { (c) } 2018 \text { The Author(s). Published by S. Karger AG, Basel } \\
\text { www.karger.com/cpb }\end{array}$ \\
\hline
\end{tabular} \\ Zou et al.: miR-4633-5p in Metastatic Melanoma}

\section{Cell culture}

As far as we know, sinonasal mucosal melanoma cell line has not been established yet. Moreover, in order to find out whether miR-4633-5p is a broad-spectrum tumor suppressor in melanoma, in vitro functional studies were carried out in two non-mucosal melanoma cell lines. Human melanoma cell lines A375 and MDA-MB-435S (M435S) were purchased from Cell Bank of Chinese Academy of Sciences (Shanghai, China). A375 were derived from cutaneous melanoma and M435S from pleural effusion of a patient with breast cancer and was therefore previously described as ductal carcinoma, but was later confirmed to be of melanoma origin [16]. A375 cells and M435S cells were cultured in high-glucose DMEM (Gibco, Shanghai, China) supplemented with 10\% FBS (Gibco).

\section{Transfection of oligo RNA}

The mature miR-4633-5p is 20nt long (full sequence: 5'-AUAUGCCUGGCUAGCUCCUC-3', miRBase accession number: MIMAT0019689) [17]. Double strand miR-4633-5p mimics, mimics control (mimics-NC), single strand anti-miR-4633-5p (anti-miR) and anti-miR control (anti-NC) were synthesized (GenePharma, Shanghai, China).

A375 cells and M435S cells were transfected with miR-4633-5p mimics and anti-miR to up- and down-regulate the expression of miR-4633-5p. A375 and M435S cells were seeded 16-24 hours prior to transfection to yield $30-50 \%$ confluence at the time of transfection. Liposomal cocktails with miR4633-5p mimics (50nM final), anti-miR (50nM final), mimics-NC (100nM final) and anti-NC (100nM final) were generated with Lipofectamine 2000 (ThermoFisher Scientific) serum-free medium following manufacturer's protocols. Cells were incubated with transfection complexes for 4-6 h prior to media change. Transfected cells were incubated for $48 \mathrm{~h}$ prior to assay seeding. RT-qPCR was carried out in parallel to confirm successful transfection.

\section{Transfection of plasmid}

Green fluorescent protein (GFP)-expressing, blasticidin-resistant plasmids carrying either shorthairpin DNA (control plasmid) or full sequence of precursor miRNA (hsa-mir-4633, mir-4633) along with $\sim 200 \mathrm{nt}$ flanking sequences were constructed. Melanoma cell seeding was performed as described above. Plasmids were transfected at a final concentration of $1 \mu \mathrm{g} / \mathrm{ml}$ with Lipofectamine 2000 (ThermoFisher Scientific) following manufacturer's protocols. After GFP was observed in melanoma cells under fluorescence microscope, $10 \mu \mathrm{g} / \mathrm{ml}$ of blasticidin (Sigma-Aldrich, Shanghai, China) was added to the culture medium and incubated for 5-7 days prior to assay seeding. RT-qPCR was carried out in parallel to confirm successful transfection.

\section{Cell proliferation assays}

Cell Counting Kit-8 (CCK-8, Dojindo, Shanghai, China) assay was carried out in 3 biological repeats, each with 4-5 technical repeats, following manufacturer's protocol. OD value at $450 \mathrm{~nm}$ was converted into cell number according to standard curves and then into fold change.

Colony formation assays were carried out in 3 biological repeats, each with 2 technical repeats. Approximately 500 melanoma cells transfected with oligo RNA or 1, 000 melanoma cells transfected with plasmid were seeded into each well of a 6-well plate (Corning, Shanghai, China) and grown for 6-8 days. Oligo RNA transfection was repeated at day 3 (upon seeding into 6-well plate) to maintain the effect. Then, the 6-well plate was washed 3 times with PBS, fixed in methanol for $5 \mathrm{~min}$, washed 3 times with distilled water, stained with $0.1 \%$ crystal violet for $10 \mathrm{~min}$, washed by distilled water, air dried and photographed.

\section{Transwell assay}

Matrigel (Corning) preparation and coating of upper chamber were performed according to manufacturer's protocol. Briefly, $2 \times 10^{4}$ melanoma cells were seeded into the upper chamber and were allowed to attach overnight before change of culture media. Then, serum-free media and culture media supplemented with 10\% FBS were added to the upper and lower chambers respectively. After 24 hours, melanoma cells that had invaded through the permeable membrane (pore diameter: $8.0 \mu \mathrm{m}$ ) were fixed in methanol and stained with crystal violet. Cells were counted under the microscope at $100 \times$ magnification. Six fields ( 3 peripheral and 3 central) were randomly chosen for cell counting. All experiments were carried 


\section{Cellular Physiology Cell Physiol Biochem 2018;49:1364-1379 and Biochemistry \begin{tabular}{l|l} 
DOI: $10.1159 / 000493414$ \\
Published online: 12 September, 2018
\end{tabular} \\ Zou et al.: miR-4633-5p in Metastatic Melanoma}

out in 3 biological repeats, each with 2 technical repeats. Percent invasion was determined using the following formula according to manufacturer's protocol:

$$
\text { invasion } \%=\frac{\text { mean number of cells invading through coated membrane }}{\text { mean number of cells invading through uncoated membrane }} \times 100
$$

\section{Gelatin zymography}

Conditioned media was prepared as previously described [18]. Gelatin zymography was carried out following manufacturer's protocol (Abcam, Shanghai, China). Briefly, $2 \times 10^{5}$ cells were seeded into each well of a 6-well plate. Upon reaching $95 \%$ confluence, culture media was changed into serum-free media and was collected after 24 hours. Culture media was then concentrated and loaded onto 7.5\% SDS-PAGE gel containing $0.1 \%$ gelatin and then electrophoresed at $4^{\circ} \mathrm{C}$ Then, gels were washed in $2.5 \%$ Triton X-100 at room temperature for $1 \mathrm{~h}$, incubated at $37^{\circ} \mathrm{C}$ for $24 \mathrm{~h}$ in incubation buffer, stained with Coomassie Brilliant Blue R-250 and destained. MMP2 activity presented as clear bands around 64kD. All experiments were carried out in 3 biological repeats, each with 2 technical repeats.

\section{Western blot analysis}

Western blot analysis was performed following manufacturer's protocols and as previously described with the following modifications for the analysis of MMP2 [6]. Conditioned cell-culture media (3 $\mu$ g proteins) was separated by 7.5\% SDS-PAGE. Blocking solution consisted of 5\% BSA (Sigma-Aldrich) in $25 \mathrm{mM}$ Tris$\mathrm{HCl}(\mathrm{pH} 7.4$ ), $150 \mathrm{mM} \mathrm{NaCl}, 3 \mathrm{mM} \mathrm{KCl}$, and 0.1\% Tween 20. Primary antibodies of MMP2, MMP9, $\beta$-actin, Akt, phospho-Akt Ser473 (pAktS473), phospho-Akt Thr308 (pAktT308), GSK-3 $\beta$, phospho-GSK-3 $\beta$ Ser9 (pGSK3ßS9), PTEN, phospho-PTEN Ser380 (pPTENS380), PDK1 and phospho-PDK1 Ser241 (pPDK1S241) were purchased (Cell Signaling Technology, Shanghai, China). All experiments were carried out in 3 biological repeats, each with 2 technical repeats. Quantification of intensity of target bands was performed by subtracting regional background and then normalizing against intensity of control bands.

\section{Statistical analysis}

Statistical analyses were performed using the SPSS software (version 19). The Kaplan-Meier analysis and log-rank test was used to assess univariate in survival analysis. The Cox regression analysis was used to assess multivariate in survival analysis. The Fisher's exact test was used to assess correlation between categorical data. The Student's $t$ test and one-way ANOVA-Bonferroni test were used to assess continuous data. Logistic regression analysis was used to assess multivariate in metastasis analysis [19]. All tests were two tailed. $\mathrm{P}<0.05$ was considered statistically significant.

\section{Results}

\section{Clinicopathologic features and survival of sinonasal mucosal melanoma}

The clinicopathologic features and DSS of all the 56 cases were summarized in Table 1. The average age at primary diagnosis was 60 years (range, 26 years to 85 years). Thirty-one $(55.4 \%)$ patients underwent endoscopic resection, 17 (30.4\%) patients underwent lateral rhinotomy, $5(8.9 \%)$ patients underwent maxillectomy and $2(3.6 \%)$ patients underwent radical maxillectomy extending to the orbit. One patient presented with cervical lymph node involvement at the time of diagnosis and therefore underwent neck dissection in addition to lateral rhinotomy. Clean surgical margin was achieved in all but two cases. For the two cases with positive margin, one patient underwent endoscopic resection and survived for 15 months; the other patient underwent maxillectomy and survived for 29 months.

In terms of adjuvant therapy, none of the patients received targeted therapy. Among the $27(48.2 \%)$ patients who received adjuvant therapy, 14 were treated in our hospital as follows. One patient received preoperative chemoradiotherapy (radiotherapy dosage: $48 \mathrm{~Gy}$; chemotherapy: dacarbazine $220 \mathrm{mg} / \mathrm{m}^{2}$ for 5 days, repeated after 3 weeks, 3 cycles in total) due to unresectable primary tumor mass. The rest 13 patients received postoperative threedimensional image-based treatment planning and intensity-modulated radiation therapy for about 7 weeks (mean dosage: $63.1 \mathrm{~Gy}$, range: 48 Gy to $71 \mathrm{~Gy}$ ). One of the 13 patients 


\section{Cellular Physiology $\quad$ Cell Physiol Biochem 2018;49:1364-1379 \begin{tabular}{l|l|l} 
DOI: 10.1159/000493414 & $\begin{array}{l}\text { O 2018 The Author(s). Published by S. Karger AG, Basel } \\
\text { www.karger.com/cpb }\end{array}$ \\
\hline
\end{tabular}

also received chemotherapy (dacarbazine $220 \mathrm{mg} / \mathrm{m}^{2}$ and cisplatin $25 \mathrm{mg} / \mathrm{m}^{2}$, repeated after 3 weeks, 4 cycles in total). As for the 13 patients who refused to receive adjuvant therapy in our hospital, two of them were treated with traditional Chinese medicine, one with preoperative radiotherapy (due to presence of cervical lymph node metastasis), two with preoperative chemoradiotherapy (due to unresectable primary tumor), three with postoperative radiotherapy and five with postoperative chemoradiotherapy. Detailed adjuvant therapy protocols were not available for these 13 patients.

In the majority of cases, surgically removed tissue was fragmented and lacking anatomic landmarks. Therefore, tumor thickness and ulceration status were not assessable. The estimated tumor size ranged from $2 \times 1 \times 0.5 \mathrm{~cm}^{3}$ to $5 \times 4.5 \times 4.0$ $\mathrm{cm}^{3}$. Three (5.4\%) patients presented with neural symptoms ( 2 with facial numbness and 1 with mild headache). However, perineural invasion was not observed microscopically. Cellular morphology of sinonasal mucosal melanoma included epithelioid, plasmacytoid, spindled, undifferentiated and mixed types $[1,19]$. Cellular morphology didn't correlate with DSS (data not shown). The most commonly observed growth pattern was perivascular growth, which was observed in $21(37.5 \%)$ cases. Bone invasion was observed in $6(10.7 \%)$ cases and didn't correlate with DSS or metastasis due to limited sample size (data not shown).

Among the clinicopathologic parameters examined in univariate survival analysis, elder age, absence of melanin and presence of metastasis (either regional or distant) were associated with shorter DSS of sinonasal mucosal melanoma, while sex, mitotic index, necrosis and treatment modality were not (Table 1, Fig. 1). Patients with no smoking history $(<10$ pack-year) and lower stage showed a trend towards having longer DSS, but was not statistically significant (Table 1, Fig. 1). When all the clinicopathologic parameters were entered into multivariate survival analysis, elder age and presence of metastasis remained statistically significant for poor DSS (relative risk [95\% confidence interval], 1.041 [1.008, $1.074], \mathrm{P}=0.013 ; 3.260$ [1.451, 7.323], $\mathrm{P}=0.004$, Cox regression analysis).

Expression of miR-4633-5p was lower in metastatic sinonasal mucosal melanoma

To find potential biomarkers for metastatic sinonasal mucosal melanoma that is independent of prognosis-associated clinicopathologic parameters (e.g. age), we evaluated 


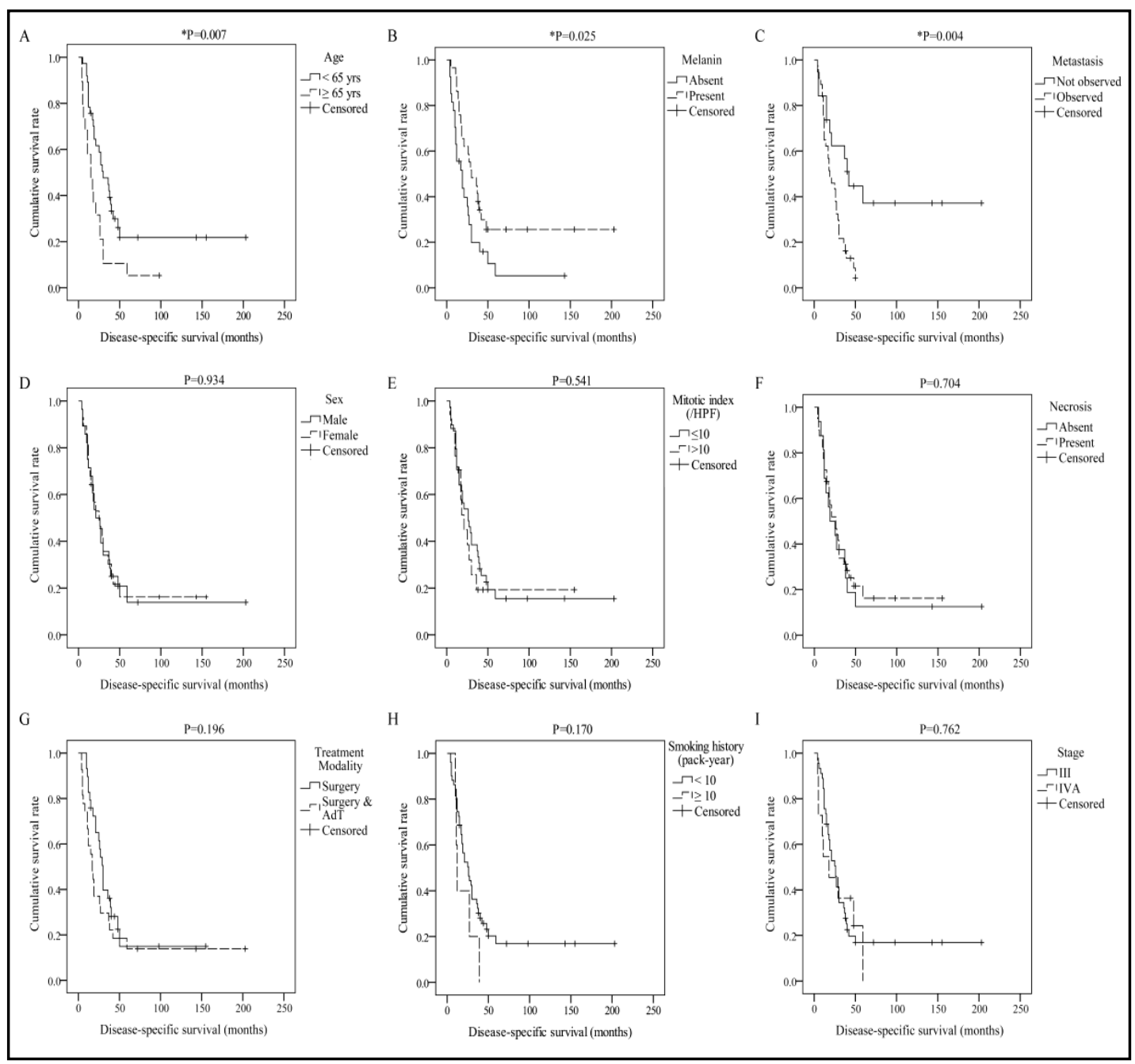

Fig. 1. Clinicopathologic parameters and disease-specific survival of sinonasal mucosal melanoma. KaplanMeier survival curves (log-rank test) of (A) age, (B) melanin, (C) metastasis, (D) sex, (E) mitotic index, (F) necrosis, (G) treatment modality, $(\mathrm{H})$ smoking history and (I) stage. ${ }^{*} \mathrm{P}<0.05$. HPF, high-power field; AdT, adjuvant therapy.

the expression of 2549 human miRNAs in eight paired cases (the discovery cohort, Table 2) by miRNA microarray. Two miRNAs were found up-regulated in cases with long DSS and two were down-regulated (Fig. 2A). Next, we examined the expression of the two miRNAs with the highest fold change (miR -30c-2-3p and miR-4633-5p) in larger sample size (the validation cohort) to verify the findings of the discovery cohort. One case was excluded due to poor quality of RNA. In the rest fifteen cases, the expression of miR-30c-2-3p was similar between different groups (data not shown). The expression of miR-4633-5p was also similar in cases with long and short DSS (mean $\Delta C T$, 8.06 vs. 9.29, $\mathrm{P}=0.465$, Student's $\mathrm{t}$ test). However, the expression of miR-4633-5p was, on average, 32 times lower in metastatic sinonasal mucosal melanoma than that in non-metastatic cases (mean $\Delta \mathrm{CT}, 11.01$ vs. 6.04, $\mathrm{P}<0.001$, Student's t test; Fig. 2B).

\section{Low expression of miR-4633-5p suggested high risk of metastasis}

To further substantiate the findings of the validation cohort, we examined the expression of miR-4633-5p in the application cohort. Three cases were excluded due to poor quality of RNA. As the highest $\Delta C T$ value of miR-4633-5p in the non-metastatic group in the validation cohort was 8.64, the cut-off $\Delta \mathrm{CT}$ value was set at 9 to minimize false positivity in the 
application cohort. Therefore, $\Delta \mathrm{CT}>9$ was regarded as miR-4633-5 $\mathrm{p}^{\text {low }}$ and $\Delta \mathrm{CT} \leq 9$ as miR$4633-5 \mathrm{p}^{\text {high }}$. In univariate analysis, miR-4633-5 $\mathrm{p}^{\text {low }}$ was able to identify metastatic sinonasal mucosal melanoma with specificity of $100 \%(5 / 5)$ and sensitivity of $87.5 \%(21 / 24)$. Area under ROC curve was 0.88 (95\% confidence interval: $0.757-1.000, \mathrm{P}=0.008$, Fig. 2C).

Next, we retrospectively reviewed the clinicopathologic features of the above 52 cases, whose miR-4633-5p status was available, to see whether miR-4633-5p ${ }^{\text {low }}$ correlated with

Table 2. Clinicopathologic features and DSS of the discovery cohort. Abbreviations: DSS, disease-specific survival; M, male; F, female; DOD, died of disease

\begin{tabular}{|c|c|c|c|c|c|c|c|c|c|c|}
\hline Pair ID & $\begin{array}{c}\text { DSS } \\
\text { (months) }\end{array}$ & Living status & Age & Sex & Metastasis & Stage & $\begin{array}{l}\text { Extent of } \\
\text { primary } \\
\text { tumor }\end{array}$ & Treatment modality & Melanin & $\begin{array}{l}\text { Smoking } \\
\text { history }\end{array}$ \\
\hline G1 & 203 & Alive & 41 & M & None & III & $\begin{array}{c}\text { Unilateral } \\
\text { nasal cavity }\end{array}$ & $\begin{array}{l}\text { Surgery \& postoperative } \\
\text { chemoradiotherapy }\end{array}$ & Present & None \\
\hline P1 & 12 & DOD & 44 & $\mathrm{~F}$ & Yes & III & $\begin{array}{l}\text { Unilateral } \\
\text { nasal cavity } \\
\text { Unilateral }\end{array}$ & $\begin{array}{l}\text { Surgery \& preoperative } \\
\text { radiotherapy }\end{array}$ & Absent & None \\
\hline G2 & 155 & Alive & 56 & $\mathrm{~F}$ & None & III & $\begin{array}{l}\text { nasal cavity } \\
\text { \& ethmoid } \\
\text { sinus }\end{array}$ & Surgery & Present & None \\
\hline P2 & 11 & DOD & 56 & M & Yes & III & $\begin{array}{l}\text { Unilateral } \\
\text { nasal cavity } \\
\text { \& ethmoid } \\
\text { sinus }\end{array}$ & $\begin{array}{l}\text { Surgery \& postoperative } \\
\text { radiotherapy }\end{array}$ & Absent & Yes \\
\hline G3 & 143 & Alive & 52 & $\mathrm{~F}$ & None & III & $\begin{array}{l}\text { Unilateral } \\
\text { nasal cavity } \\
\text { \& ethmoid } \\
\text { sinus \& } \\
\text { maxillary } \\
\text { sinus }\end{array}$ & $\begin{array}{l}\text { Surgery \& postoperative } \\
\text { radiotherapy }\end{array}$ & Absent & None \\
\hline P3 & 12 & DOD & 56 & M & Yes & III & $\begin{array}{c}\text { Unilateral } \\
\text { nasal cavity }\end{array}$ & $\begin{array}{l}\text { Surgery \& postoperative } \\
\text { radiotherapy }\end{array}$ & Present & None \\
\hline G4 & 98 & Alive & 71 & $\mathrm{~F}$ & None & III & $\begin{array}{c}\text { Unilateral } \\
\text { nasal cavity }\end{array}$ & Surgery & Present & None \\
\hline P4 & 6 & DOD & 74 & $\mathrm{~F}$ & Yes & III & $\begin{array}{c}\text { Unilateral } \\
\text { nasal cavity }\end{array}$ & $\begin{array}{c}\text { Surgery \& postoperative } \\
\text { radiotherapy }\end{array}$ & Absent & None \\
\hline
\end{tabular}

Fig. 2. miR-4633-5p was differentially expressed in metastatic and nonmetastatic sinonasal mucosal melanoma. (A) Representative miRNAs differentially expressed in patients with long $(G, \geq 36$ months) and short ( $\mathrm{P}, \leq 12$ months) disease-specific survival in the discovery cohort by miRNA microarray assay. Asterisks indicate the 4 miRNAs that were differentially expressed in all 4 pairs of cases. (B) Expression of miR-4633-5p in the validation cohort by quantitative reverse-transcriptase PCR analysis. (C) Receiver operating characteristic (ROC) curve of miR-4633-5p in the application cohort.

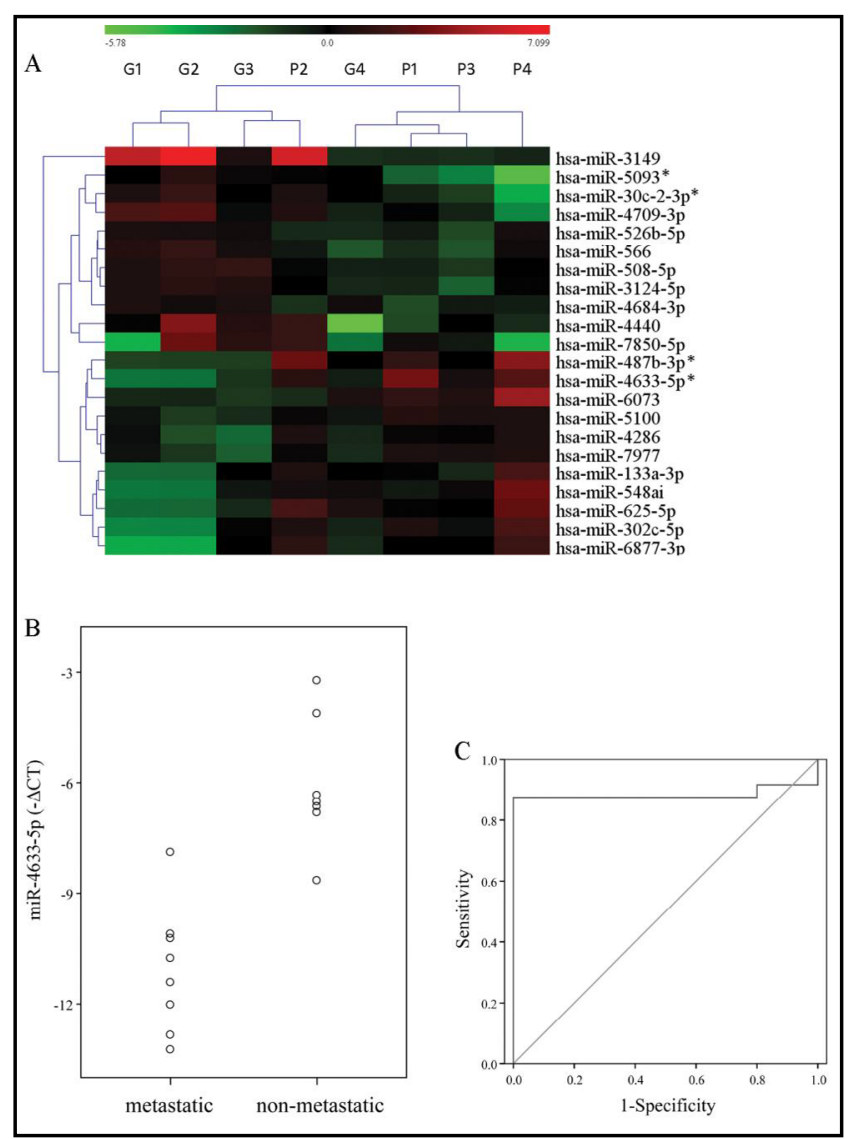




\section{Cellular Physiology Cell Physiol Biochem 2018;49:1364-1379

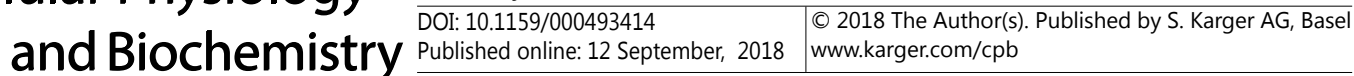 \\ Zou et al.: miR-4633-5p in Metastatic Melanoma}

other invasive features. When all the available clinicopathologic parameters and miR-4633$5 p$ status were entered into multivariate Logistic regression analysis, miR-4633-5p $p^{\text {low }}$ was the only factor that associated with metastasis (relative risk [ $95 \%$ confidence interval], 54.22 [6.01, 489.45], $\mathrm{P}<0.001)$. Further statistical analyses suggested that miR-4633-5p status didn't correlate with any of the available clinicopathologic parameters (data not shown).

\section{Overexpression of miR-4633-5p suppressed growth of melanoma cells in vitro}

To investigate whether miR-4633-5p functions as tumor suppressor in melanoma cells, in vitro functional studies were carried out. CCK-8 assay and colony formation assay were carried out to explore whether miR-4633-5p suppressed cell proliferation and colony formation. M435S cells transfected with miR-4633-5p mimics showed significantly decreased proliferation and colony formation than those transfected with mimics-NC, while the application of anti-miR failed to exhibit any difference in both CCK-8 assay and colony formation assay (Fig. 3A-B). Parallel RT-qPCR demonstrated that expression of miR-4633$5 p$ changed over 2 -fold upon transfection (data not shown). Experiments with A375 cells showed similar results (data not shown). We reckoned that the seemingly ineffectiveness of miR-4633-5p knockdown was due to the low basal expression of miR-4633-5p in melanoma cell lines, which was highly likely to be far below threshold (mean $\Delta$ CT, 11.1 for M435S and 14.8 for A375).

\section{Knockdown of miR-4633-5p promoted growth of melanoma cells in vitro}

To verify our hypothesis and to mimic endogenous maturation of miR-4633-5p, A375 cells and M435S cells were transfected with mir-4633-expressing plasmid or control plasmid. As expected, A375 cells transfected with mir-4633 showed decreased proliferation and colony formation than those transfected with control plasmid (Fig. 3C-D). Moreover, the application of anti-miR reversed the growth-inhibitory effect of mir-4633, while the application of anti-NC resulted in minimal difference (Fig. 3C-D). Parallel RT-qPCR also demonstrated that although the expression of miR-4633-5p and miR-4633-3p both increased by $2^{8}$ fold upon transfection of mir-4633, the application of anti-miR down-regulated exclusively the expression of miR-4633-5p by $2^{4}$ fold, while the expression of miR-4633$3 p$ remained unchanged. Experiments with M435S cells showed similar results (data not shown). Therefore, overexpression of miR-4633-5p suppressed growth of melanoma cells in vitro, while knockdown of miR-4633-5p promoted cell growth.

Overexpression of miR-4633-5p suppressed invasiveness and secretion of MMP2 in A375 cells in vitro

To investigate whether miR-4633-5p regulates the migratory activity of melanoma cells in vitro, un-coated and coated transwell assays were performed. A375 cells transfected with mir-4633-expressing plasmid showed decreased invasiveness than cells transfected with control plasmid (mean invasion\%, 50.7 vs. 83.0, $\mathrm{P}=0.001$, one-way ANOVA-Bonferroni test, Fig. 4A). Moreover, the application of anti-miR reversed the invasiveness-inhibitory effect of mir-4633, while the application of anti-NC resulted in minimal difference (mean invasion\%, 76.9 vs.48.7, $\mathrm{P}=0.006$, one-way ANOVA-Bonferroni test, Fig. 4A). Repeated experiments with M435S cells failed to yield any result, as none of the cells invaded through the coated permeable membrane (data not shown).

MMP2 and MMP9 are two of the major metalloproteinases that play a crucial role in extracellular matrix remolding [20]. To explore whether the status of these two enzymes were altered upon overexpressing miR-4633-5p in melanoma cells, we evaluated the expression of MMP2 and MMP9 in A375 cells and M435S cells. Results of both RT-qPCR and western blot analysis of cell lysate showed that the expressions of MMP9 in both cell lines were below detection limit. Meanwhile, the expression of MMP2 in M435S cells was also below detection limit (data not shown), which was in accordance with data in the Cancer Cell Line Encyclopedia database (CCLE, www.broadinstitute.org/ccle) and was able to explain why M435S cells failed to invade through the coated permeable membrane in the above 


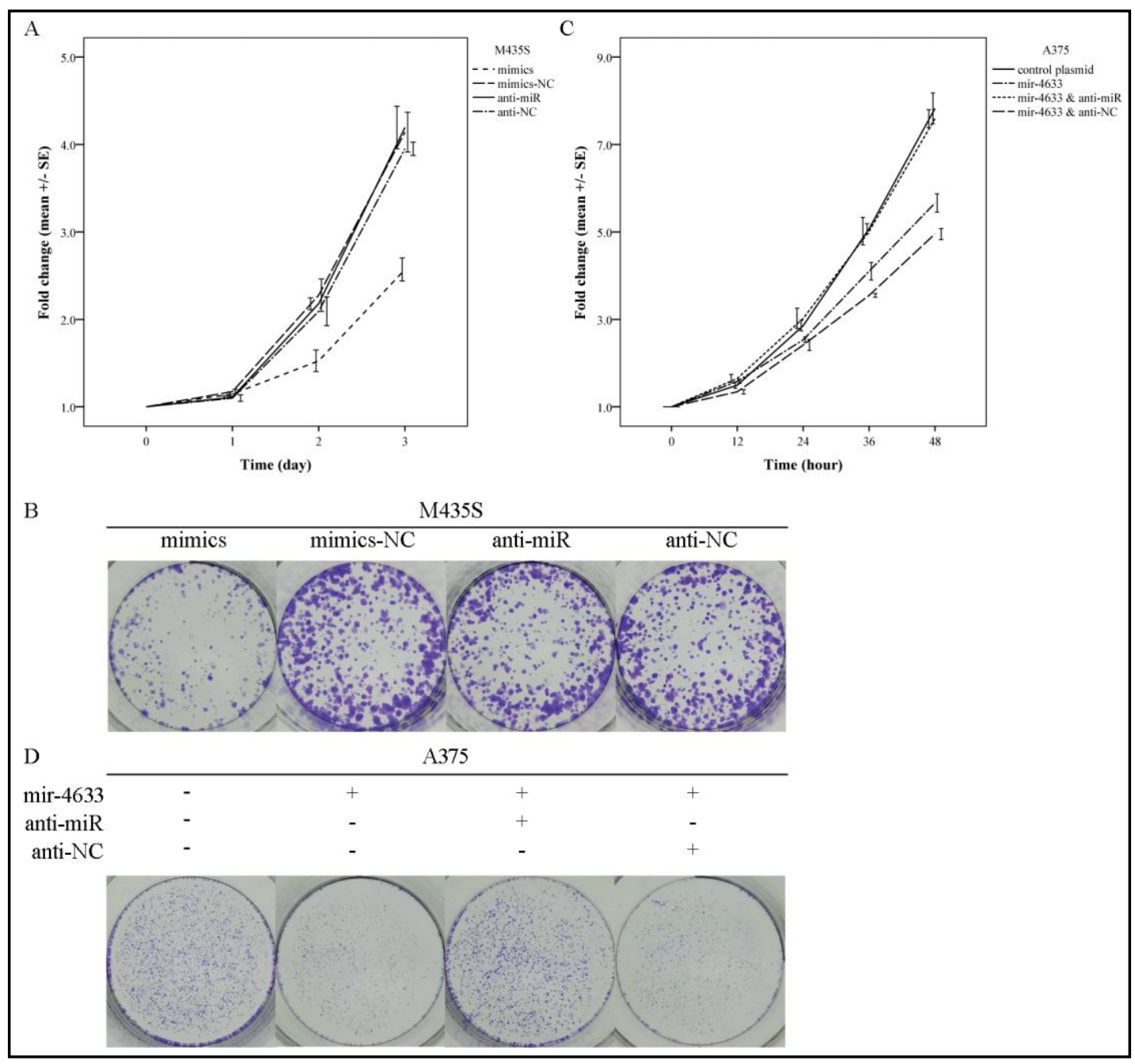

Fig. 3. Overexpression of miR-4633-5p inhibits proliferation and colony formation of melanoma cells in vitro, and vice versa. (A) CCK-8 assay of M435S cells transfected with miR-4633-5p mimics, mimics-NC, antimiR and anti-NC. (B) Approximately 500 M435S cells were transfected with miR-4633-5p mimics, mimicsNC, anti-miR and anti-NC and were stained at day 8. (C) CCK-8 assay of A375 cells transfected with control plasmid, mir-4633, mir-4633 \& anti-miR and mir-4633 \& anti-NC. (D) Approximately 1000 A375 cells were transfected with control plasmid, mir-4633, mir-4633 \& anti-miR and mir-4633 \& anti-NC and were stained at day 6.

experiments. Therefore, only the secretion of MMP2 in A375 cells was further evaluated.

Both gelatin zymography and western blot analysis of culture media showed that A375 cells transfected with mir-4633-expressing plasmid secreted less pro-MMP2 and MMP2 than those transfected with control plasmid (Fig. 4B). In addition, the application of anti-miR reversed the secretion-inhibitory effect of mir-4633, while the application of anti-NC resulted in minimal difference (Fig. 4B). To further explore whether the decrease in secretion was caused by reduced synthesis of MMP2, we evaluated the expression of MMP2 in A375 cell lysate. Both RT-qPCR and western blot analysis showed that endogenous expression of MMP2 mRNA and protein remained unchanged in A375 cell lysate (Fig. 4C-D). Therefore, overexpressing miR-4633-5p suppressed the invasiveness and secretion, but not transcription or translation, of MMP2 in A375 cells in vitro. 


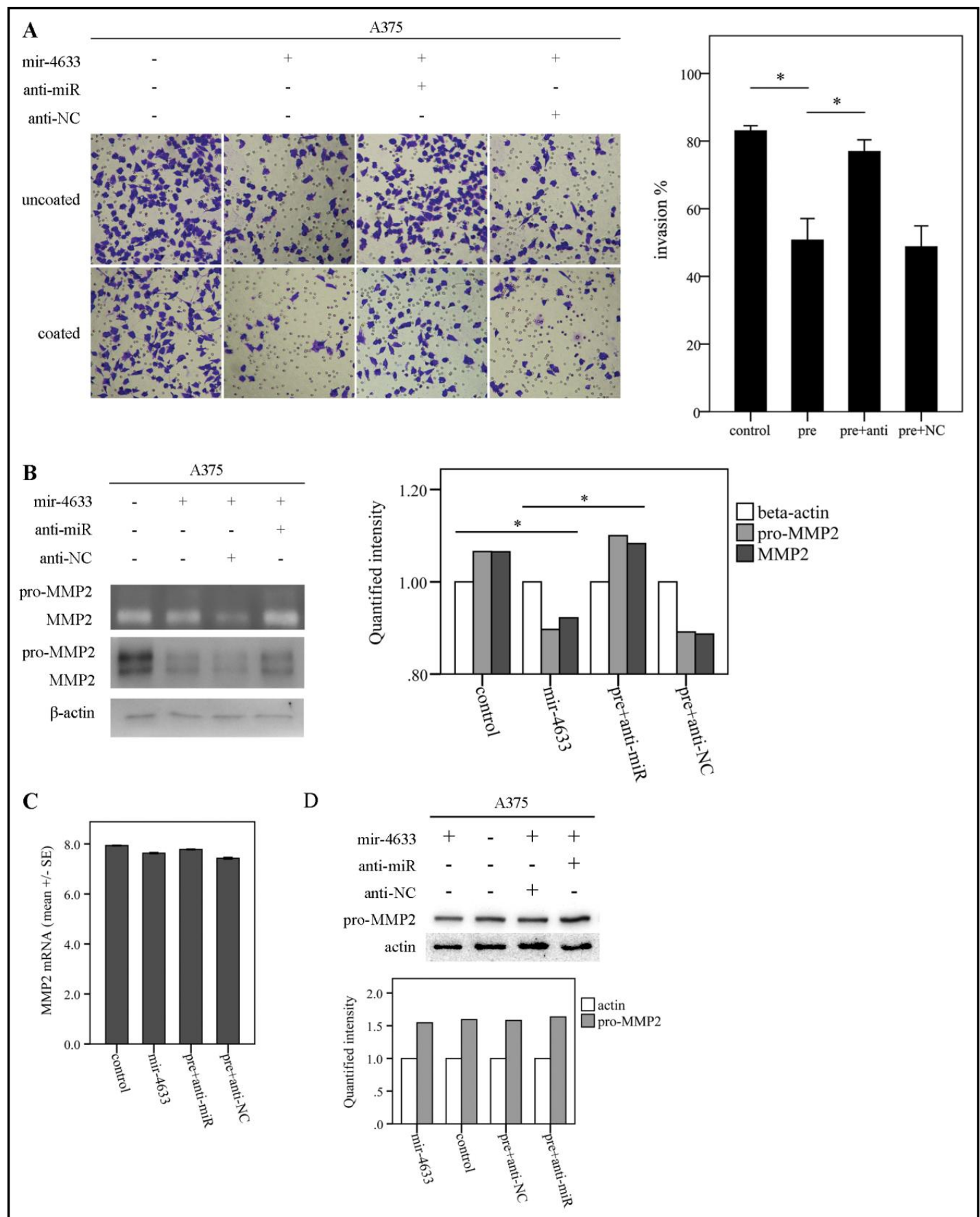

Fig. 4. miR-4633-5p inhibits invasiveness of and secretion of MMP2 of A375 cells in vitro. (A) Representative images and invasion \% (mean +/- SE) of uncoated and coated transwell assays of A375 cells. Pore diameter: $8.0 \mu \mathrm{m}$. (B) Representative images of gelatin zymography (bright bands) and western blot (dark bands) of MMP2 secreted by A375 cells. (C) Representative result of MMP2 mRNA level ( $\triangle$ CT value) in A375 cell lysate by qRT-PCR. (D) Representative images of western blot of MMP2 in A375 cell lysate. *Statistically significant. Abbreviations: SE, standard error; control, control plasmid; pre, mir-4633; pre+anti, mir-4633 \& anti-miR; pre+NC, mir-4633 \& anti-NC. 
Overexpression of miR4633-5p inactivated Akt pathway in vitro

Activation of Akt pathway was reported to promote survival and metastasis of melanoma cells [12, 13]. Therefore, we evaluated the phosphorylation status of Akt, its upstream regulators (PDK1 and PTEN) and its downstream target (GSK$3 \beta$ ) upon overexpression of miR-4633-5p [12, 13]. In both A375 cells and M435S cells, cells transfected with mir-4633-expressing plasmid showed decreased expression of pAktS473, pAktT308, pPDK1S241, pPTENS380 and pGSK3ßS9 compared with those transfected with control plasmid (Fig. 5). Moreover, the application of anti-miR restored phosphorylation of Akt, PDK1, PTEN and GSK-3 $\beta$ compared with that of anti-NC (Fig. 5). Therefore, overexpression of miR-4633-5p induced inactivation of Akt pathway in melanoma cells in vitro.

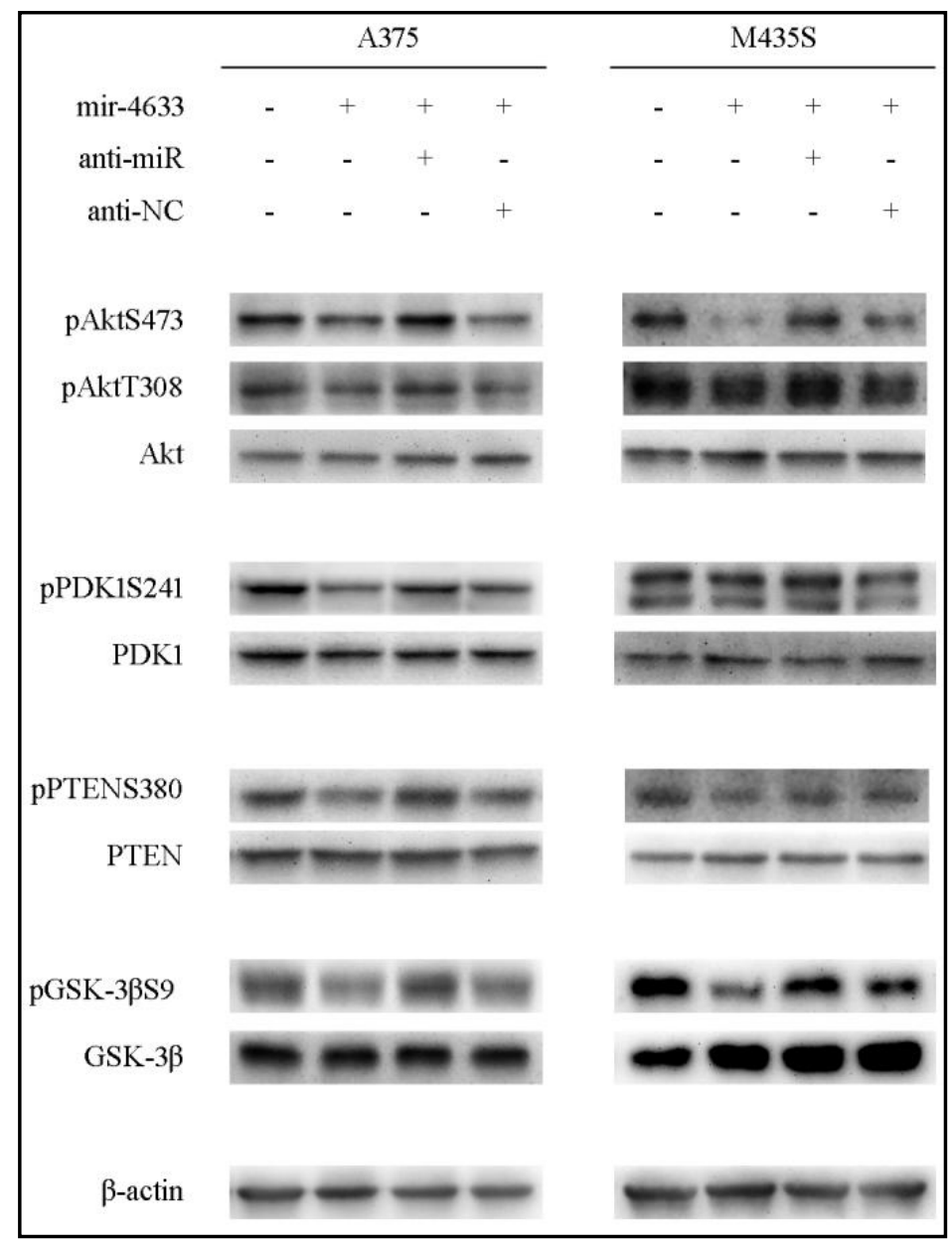

Fig. 5. miR-4633-5p inhibits activation of Akt signaling pathway in melanoma cells in vitro. Western blot analysis of phosphorylation status of pAktS473, pAktT308, pPDK1S241, pPTENS380 and pGSK$3 \beta S 9$ in melanoma cells in vitro.

\section{Discussion}

With the growing knowledge about the biology of miRNAs, especially the stem-loop structure of miRNA precursor, new miRNAs are continuously being discovered, among which is miR-4633-5p. However, since its discovery, the role of miR-4633-5p in carcinogenesis has not been studied yet [21]. Our study demonstrates for the first time that low expression of miR-4633-5p accurately discriminates metastatic sinonasal mucosal melanoma patients from non-metastatic patients. Additionally, miR-4633-5p suppresses the aggressive growth of melanoma cells through inhibition of MMP2 secretion and inactivation of Akt pathway.

MiRNAs were found to be rather stable in FFPE sample over years. This durable merit of miRNA permits us to retrospectively screen the miRNA profile in rare types of melanoma. The present study is the first time to explore miRNA expression profile of melanoma in sinonasal mucosa, while the previous researches studying miRNA expression profile either in melanoma tissue or in plasma mainly focused on cutaneous melanoma, uveal melanoma and conjunctival melanoma. So far, dozens of miRNAs were found to associate with pathogenesis, prognosis and differential diagnosis of melanoma [8, 9, 22-24]. For example, a panel of 4 miRNAs (miR-150-5p, miR-15b-5p, miR-16-5p and miR-374b-3p), when combined with stage, distinguish melanomas with brain metastasis from non-metastatic patients [22]. 


\section{Cellular Physiology Cell Physiol Biochem 2018;49:1364-1379

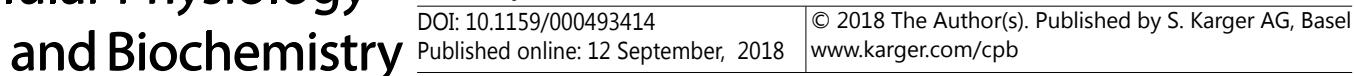 \\ Zou et al.: miR-4633-5p in Metastatic Melanoma}

Additionally, two circulating miRNAs (miR-1246 and miR-185) were found to discriminate patients with metastatic melanoma from healthy subjects with sensitivity of $90.5 \%$ and specificity of $89.1 \%$ [23]. Apart from findings of miRNAs as biomarkers in the metastatic course of melanoma, a number of clinical trials using miRNAs as therapeutic means for cancer treatment have been carried out and some preliminary data have shown inspiring outcomes [24].

MiRNAs affect multiple cellular functions via regulating a myriad of downstream targets that relate to tumorigenesis. The aberrant activation of the Akt signaling pathway is extensively involved in the development of melanoma $[12,13]$. Previous studies showed that the expression of p-AktSer473 was higher in melanoma than in nevi and that positive immunohistochemical staining of p-AktSer473 was associated with worse 5-year survival in primary melanoma $[25,26]$. In Akt signaling pathway, PDK1 phosphorylates and activates Akt at the cell membrane while PTEN serves as an off-switch [27]. The phosphorylation of PTEN suppresses its phosphatase activity, which in turn facilitates the recruitment of Akt to the cell membrane, where Akt is activated [28]. The activated Akt then exerts its functions through phosphorylation of numerous downstream targets, including GSK-3 $\beta$, that relate to cell proliferation and migration. Here we found that proliferation of melanoma cells and activation of Akt pathway changed conversely with the expression of miR-4633-5p, suggesting that miR-4633-5p negatively regulates the growth of melanoma cells through inactivation of Akt pathway.

Regardless of site of origin, invading through the degraded extracellular matrix is crucial for melanoma cells to leave primary site and to form metastases. MMP2 has long been shown to participate in extracellular matrix remodeling and metastasis of melanoma [20, 29]. MMP2 is synthesized in the cytoplasm as an inactive pro-enzyme (pro-MMP2) and then secreted into the extracellular matrix, where pro-MMP2 is recruited to the cell membrane and cleaved into the active form (MMP2) [30]. Once activated, MMP2 degrades gelatin and other extracellular components, which in turn facilitates metastasis and growth of melanoma cells [29]. In the present study, invasiveness of melanoma cells and secretion of MMP2 in melanoma cells changed conversely with the expression of miR-4633-5p, suggesting the inhibitory role of miR-4633-5p on metastasis was mediated by reducing the secretion of MMP2. Interestingly, we found that while transcription and translation of MMP2 remained unchanged, only the secretory process of MMP2 was affected by miR-4633-5p, which is in part in contrast to previous studies, showing that secreted MMP2 changed in concert with MMP2 mRNA and cytoplasmic pro-MMP2 [30]. This finding was repeatedly verified by means of gelatin zymography, western blot analysis and RT-qPCR, and all results led to the conclusion that miR-4633-5p inhibits exclusively the secretory process, but neither at the transcriptional nor at the translational levels of MMP2, through intricate mechanisms which we have not yet understood.

In the present study, in vivo transplantation experiments were not included because of diminishing expression of miR-4633-5p in transfected cells. Repeated experiments showed that the expression level of miR-4633-5p in melanoma cells transfected with either mir-4633expressing plasmid or mir-4633-expressing lentiviral vectors (data not shown) diminished over a period of 2-3 weeks in culture, while GFP expression and blasticidin/puromycinresistance were both observed in nearly $100 \%$ of cells, indicating the functional presence of the transfected exogenous genes. While the reason remains unknown, we speculate that it may reflect the growth advantage of cells with lower expression of miR-4633-5p that dominates the majority of the positive cell clones after prolonged cultivation [31]. It is possible that, after being transfected with mir-4633-expressing lentivirus, which was supposed to generate stable miR-4633-5p-overexpressing cells, the cells acquired merely blasticidin/puromycin-resistance but no intensive overexpression of miR-4633-5p. These cells cannot be eliminated by blasticidin/puromycin and eventually outgrow the miR-4633$5 p$-overexpressing cells. Another possible explanation is that there is negative feedback of miR-4633-5p in melanoma cells through mechanism that still remains elusive [32]. 


\section{Cellular Physiology Cell Physiol Biochem 2018;49:1364-1379 \begin{tabular}{l|l|l} 
and Biochemistry & Dublished online: 12 September, 2018 & $\begin{array}{l}\text { O } 2018 \text { The Author(s). Published by S. Karger AG, Basel } \\
\text { www.karger.com/cpb }\end{array}$ \\
\hline
\end{tabular}

In situ quantification of miR-4633-5p may help bring our findings from the bench to the bedside. Clinical trials on targeted therapy, especially on immunotherapy, have shed light on the treatment of advanced-stage cutaneous melanoma, but less on patients with sinonasal mucosal melanoma [33]. To certain extent, it is due to the low incidence of sinonasal mucosal melanoma among melanomas of all sites [1]. Additionally, as was observed in 4 cases in our study ( 1 in the validation cohort and 3 in the application cohort), heavily pigmented (melanin present in $>60 \%$ melanoma cells) melanoma tissue often yield poor-quality RNA that caused failure of cDNA amplification in RT-qPCR analysis. In practice, however, heavily pigmented melanoma takes up a considerable amount of sinonasal mucosal melanoma cases [19]. Moreover, small biopsy samples and samples with acute inflammation often fail to provide adequate high-purity tumor tissue for RNA extraction (observed in 3 cases in our study), calling for in situ evaluation of miRNA. However, in situ detection of miRNA is presently challenged by technical limitations as even the newly developed RNAscope technology is unable to detect RNAs shorter than 35nt in situ.

\section{Conclusion}

In conclusion, our finding suggests that miR-4633-5p may not only serve as a promising predictive biomarker in metastatic sinonasal mucosal melanoma, but also function as a pivotal tumor suppressor that negatively regulates the invasive activities of melanoma cells during the course of metastasis. Quantitative detection of miR-4633-5p can identify sinonasal mucosal melanoma patients with high risk of metastasis, which, in turn, may lead to the development of more personalized treatment that eventually improves the prognosis.

\section{Acknowledgements}

We thank Dr. Shuyang Wang, Dr. Yongchang Chen and Dr. Zhaozhong Ding for language polishing of the manuscript.

\section{Disclosure Statement}

No conflict of interests exists.

\section{References}

1 Williams MD: Update from the 4th Edition of the World Health Organization Classification of Head and Neck Tumours: Mucosal Melanomas. Head Neck Pathol 2017;11:110-117.

-2 Carvajal RD, Spencer SA, Lydiatt W: Mucosal melanoma: a clinically and biologically unique disease entity. J Natl Compr Canc Netw 2012;10:345-356.

-3 Takagi M, Ishikawa G, Mori W: Primary malignant melanoma of the oral cavity in Japan. With special reference to mucosal melanosis. Cancer 1974;34:358-370.

-4 López F, Rodrigo JP, Cardesa A, Triantafyllou A, Devaney KO, Mendenhall WM, Haigentz M Jr, Strojan P, Pellitteri PK, Bradford CR, Shaha AR, Hunt JL, de Bree R, Takes RP, Rinaldo A, Ferlito A: Update on primary head and neck mucosal melanoma. Head Neck 2016;38:147-155.

5 Jonas S, Izaurralde E: Towards a molecular understanding of microRNA-mediated gene silencing. Nat Rev Genet 2015;16:421-433.

-6 Wang S, Wang L, Bayaxi N, Li J, Verhaegh W, Janevski A, Varadan V, Ren Y, Merkle D, Meng X, Gao X, Wang H, Ren J, Kuo WP, Dimitrova N, Wu Y, Zhu H: A microRNA panel to discriminate carcinomas from high-grade intraepithelial neoplasms in colonoscopy biopsy tissue. Gut 2013;62:280-289. 


\section{Cellular Physiology Cell Physiol Biochem 2018;49:1364-1379 \begin{tabular}{l|l|l} 
DOI: 10.1159/000493414 & $\begin{array}{l}\text { O 2018 The Author(s). Published by S. Karger AG, Basel } \\
\text { www.karger.com/cpb }\end{array}$ \\
\hline
\end{tabular}

7 Rosenwald S, Gilad S, Benjamin S, Lebanony D, Dromi N, Faerman A, Benjamin H, Tamir R, Ezagouri M, Goren E, Barshack I, Nass D, Tobar A, Feinmesser M, Rosenfeld N, Leizerman I, Ashkenazi K, Spector Y, Chajut A, Aharonov R: Validation of a microRNA-based qRT-PCR test for accurate identification of tumor tissue origin. Mod Pathol 2010;23:814-823.

-8 Wang K, Zhang ZW: Expression of miR-203 is decreased and associated with the prognosis of melanoma patients. Int J Clin Exp Pathol 2015;8:13249-13254.

-9 Triozzi PL, Achberger S, Aldrich W, Crabb JW, Saunthararajah Y, Singh AD: Association of tumor and plasma microRNA expression with tumor monosomy-3 in patients with uveal melanoma. Clin Epigenetics 2016;8:80.

10 Liu A, Tetzlaff MT, Vanbelle P, Elder D, Feldman M, Tobias JW, Sepulveda AR, Xu X: MicroRNA expression profiling outperforms mRNA expression profiling in formalin-fixed paraffin-embedded tissues. Int J Clin Exp Pathol 2009;2:519-527.

11 Carvajal RD, Spencer SA, Lydiatt W: Mucosal melanoma: a clinically and biologically unique disease entity. J Natl Compr Canc Netw 2012;10:345-356.

12 Galbán S, Apfelbaum AA, Espinoza C, Heist K, Haley H, Bedi K, Ljungman M, Galbán CJ, Luker GD, Dort MV, Ross BD: A Bifunctional MAPK/PI3K Antagonist for Inhibition of Tumor Growth and Metastasis. Mol Cancer Ther 2017;16:2340-2350.

13 Khosravi S, Wong RP, Ardekani GS, Zhang G, Martinka M, Ong CJ, Li G: Role of EIF5A2, a downstream target of Akt, in promoting melanoma cell invasion. Br J Cancer 2014;110:399-408.

$>14$ Ahmed OA, Kelly C: Head and neck melanoma (excluding ocular melanoma): United Kingdom National Multidisciplinary Guidelines. J Laryngol Otol 2016;130:S133-S141.

15 Spandidos A, Wang X, Wang H, Dragnev S, Thurber T, Seed B: A comprehensive collection of experimentally validated primers for Polymerase Chain Reaction quantitation of murine transcript abundance. BMC Genomics 2008;9:633.

16 Chambers AF: MDA-MB-435 and M14 cell lines: identical but not M14 melanoma? Cancer Res 2009;69:5292-5293.

17 Kozomara A, Griffiths-Jones S: miRBase: annotating high confidence microRNAs using deep sequencing data. Nucleic Acids Res 2014;42:D68-D73.

18 Yang JM, Xu Z, Wu H, Zhu H, Wu X, Hait WN: Overexpression of extracellular matrix metalloproteinase inducer in multidrug resistant cancer cells. Mol Cancer Res 2003;1:420-427.

19 Zhu W, Zou B, Wang S: Clinicopathological Features and Prognosis of Sinonasal Mucosal Malignant Melanoma: A Retrospective Study of 83 Cases in a Chinese Population. ORL J Otorhinolaryngol Relat Spec 2016;78:94-104.

20 Giannelli G, Antonaci S: Gelatinases and their inhibitors in tumor metastasis: from biological research to medical applications. Histol Histopathol 2002;17:339-345.

21 Persson H, Kvist A, Rego N, Staaf J, Vallon-Christersson J, Luts L, Loman N, Jonsson G, Naya H, Hoglund M, Borg A, Rovira C: Identification of new microRNAs in paired normal and tumor breast tissue suggests a dual role for the ERBB2/Her2 gene. Cancer Res 2011;71:78-86.

-22 Hanniford D, Zhong J, Koetz L, Gaziel-Sovran A, Lackaye DJ, Shang S, Pavlick A, Shapiro R, Berman R, Darvishian F, Shao Y, Osman I, Hernando E: A miRNA-Based Signature Detected in Primary Melanoma Tissue Predicts Development of Brain Metastasis. Clin Cancer Res 2015;21:4903-4912.

23 Armand-Labit V, Meyer N, Casanova A, Bonnabau H, Platzer V, Tournier E, Sansas B, Verdun S, Thouvenot B, Hilselberger B, Doncescu A, Lamant L, Lacroix-Triki M, Favre G, Pradines A: Identification of a Circulating MicroRNA Profile as a Biomarker of Metastatic Cutaneous Melanoma. Acta Derm Venereol 2016;96:29-34.

24 Monroig-Bosque Pdel C, Rivera CA, Calin GA: MicroRNAs in cancer therapeutics: "from the bench to the bedside". Expert Opin Biol Ther 2015;15:1381-1385.

25 Kantrow SM, Boyd AS, Ellis DL, Nanney LB, Richmond A, Shyr Y, Robbins JB: Expression of activated Akt in benign nevi, Spitz nevi and melanomas. J Cutan Pathol 2007;34:593-596.

26 Dai DL, Martinka M, Li G: Prognostic significance of activated Akt expression in melanoma: a Clinicopathologic study of 292 cases. J Clin Oncol 2005;23:1473-1482.

-27 Scortegagna M, Lau E, Zhang T, Feng Y, Sereduk C, Yin H, De SK, Meeth K, Platt JT, Langdon CG, Halaban R, Pellecchia M, Davies MA, Brown K, Stern DF, Bosenberg M, Ronai ZA: PDK1 and SGK3 Contribute to the Growth of BRAF-Mutant Melanomas and Are Potential Therapeutic Targets. Cancer Res 2015;75:13991412. 


\section{Cellular Physiology Cell Physiol Biochem 2018;49:1364-1379 \begin{tabular}{ll|l} 
DOI: 10.1159/000493414 & $\begin{array}{l}\text { O 2018 The Author(s). Published by S. Karger AG, Basel } \\
\text { www.karger.com/cpb }\end{array}$ \\
\hline
\end{tabular} \\ Zou et al.: miR-4633-5p in Metastatic Melanoma}

28 Nakahata S, Ichikawa T, Maneesaay P, Saito Y, Nagai K, Tamura T, Manachai N, Yamakawa N, Hamasaki M, Kitabayashi I, Arai Y, Kanai Y, Taki T, Abe T, Kiyonari H, Shimoda K, Ohshima K, Horii A, Shima H, Taniwaki M, et al.: Loss of NDRG2 expression activates PI3K-AKT signalling via PTEN phosphorylation in ATLL and other cancers. Nat Commun 2014;5:3393.

29 Sato H, Takino T: Coordinate action of membrane-type matrix metalloproteinase-1 (MT1-MMP) and MMP-2 enhances pericellular proteolysis and invasion. Cancer Sci 2010;101:843-847.

-30 Koo BH, Kim YH, Han JH, Kim DS: Dimerization of matrix metalloproteinase-2 (MMP-2): functional implication in MMP-2 activation. J Biol Chem 2012;287:22643-22653.

-31 Turula H, Smith CJ, Grey F, Zurbach KA, Snyder CM: Competition between T cells maintains clonal dominance during memory inflation induced by MCMV. Eur J Immunol 2013;43:1252-1263.

32 Ha M, Kim VN: Regulation of microRNA biogenesis. Nat Rev Mol Cell Biol 2014;15:509-524.

-33 Robert C, Karaszewska B, Schachter J, Rutkowski P, Mackiewicz A, Stroiakovski D, Lichinitser M, Dummer R, Grange F, Mortier L, Chiarion-Sileni V, Drucis K, Krajsova I, Hauschild A, Lorigan P, Wolter P, Long GV, Flaherty K, Nathan P, Ribas A, et al.: Improved overall survival in melanoma with combined dabrafenib and trametinib. N Engl J Med 2015;372:30-39. 